\title{
Review Article \\ Spinal Epidural Abscess: A Review with Special Emphasis on Earlier Diagnosis
}

\author{
Allison Bond and Farrin A. Manian \\ Department of Medicine, Massachusetts General Hospital, Harvard Medical School, Boston, MA, USA \\ Correspondence should be addressed to Farrin A. Manian; fmanian@mgh.harvard.edu
}

Received 14 July 2016; Accepted 24 October 2016

Academic Editor: André Talvani

Copyright ( 2016 A. Bond and F. A. Manian. This is an open access article distributed under the Creative Commons Attribution License, which permits unrestricted use, distribution, and reproduction in any medium, provided the original work is properly cited.

Spinal epidural abscess (SEA) is an uncommon but serious condition with significant morbidity and mortality. The prognosis of SEA is highly dependent on the timeliness of its diagnosis before neurological deficits develop. Unfortunately, often due to its nonspecific presentation, such as back pain, the diagnosis of SEA may be delayed in up to 75\% of cases. Although many risk factors for SEA can be found in the published literature, their utility is limited by their frequent lack of objective evidence, numerousness, and absence in a significant proportion of cases. In this review, we call for a more discriminate evidence-based use of the term "risk factor" when discussing SEA and explore several approaches to its earlier diagnosis, including a simple algorithm based on its pathophysiology and serum C-reactive protein or erythrocyte sedimentation rate.

\section{Introduction}

Since its original postmortem description more than 250 years ago by Giovanni Morgagni, spinal epidural abscess (SEA) has often evaded timely diagnosis, with up to $75 \%$ of cases misdiagnosed on their initial healthcare encounter $[1,2]$. Such delays in diagnosis-and therefore of timely therapy-may lead to significant morbidity and mortality [3]. Because the signs and symptoms of SEA are often nonspecific, a high index of suspicion is key to making a timely diagnosis. The goal of this article is to provide clinicians with a general overview of SEA with a special emphasis on critical examination of its reported risk factors and exploration of potential approaches to its earlier diagnosis.

\section{Epidemiology}

2.1. Incidence. Although SEA is uncommon, its incidence is rising. From 1975 to 1998 , for example, the incidence of SEA rose from $0.2-1.2$ to $2.5-3.0$ cases per 10,000 hospital admissions [3]. This number is expected to rise further given a likely increase in the prevalence of patients at risk of SEA $[3,4]$. Heightened awareness of SEA and increased use of sensitive imaging techniques such as magnetic resonance imaging
(MRI) may also contribute to the rise in the number of reported cases [5, 6]. Although male predominance with higher prevalence between the fifth and seventh decade of life is often reported in SEA, a wide age distribution affecting virtually all age groups is also commonly described [7].

2.2. Risk Factors. Published risk factors for SEA are numerous and include diabetes mellitus (DM), intravenous drug use (IVDU), alcohol abuse, infection with human immunodeficiency virus (HIV), degenerative joint disease, recent trauma or surgery, and the presence of spinal stimulators or catheters $[3,7-9]$. Local or systemic infections are also commonly listed as risk factors; these include skin and soft tissue infections, osteomyelitis, urinary tract infection (UTI), sepsis, and indwelling vascular access infection. In addition, hypertension, chronic obstructive pulmonary disease (COPD), chronic liver or kidney disease, nerve acupuncture, tattooing, epidural analgesia, and nerve block are also thought to increase the likelihood of SEA $[8,9]$. Although reported risk factors are often intended to heighten awareness and facilitate earlier diagnosis of SEA, their routine clinical application has several limitations.

First, published risk factors or predisposing conditions for SEA are often derived from case reports, small case series, 
and literature reviews, which often fail to distinguish preexisting or potentially causative factors from coexisting conditions that may have very little role in the causation of SEA [3-15]. This is an important distinction because a risk factor must not only be shown to precede the disease but also be independently associated with its development [16-18]. For example, a frequently cited "meta-analysis" article implausibly lists several conditions such as "hepatitis," "vaginal infection," and "typhus" as risk factors or sources of infection for SEA based on descriptive case reports [7]; parenthetically, despite its title, no meta-analysis was performed [19]. Another article lists hypertension as a predisposing factor for SEA by "diminishing effective immune responses," without offering any further explanation on its mechanism [9]. Even when more plausible conditions such as degenerative spinal disease, psoas abscess, or spinal trauma are listed as risk factors, their mere presence as a coexisting condition or a complication of the SEA itself is difficult to exclude [7]. For example, with more than $80 \%$ of adults in their 50 s or older having lumbar spondylosis, a high prevalence of degenerative spinal disease in older patients with SEA would not be surprising [20] and cannot automatically be assumed to be related to this condition. Similarly, psoas abscess may not only precede SEA but also complicate it $[19,21]$. Even spinal trauma cannot be assumed to uniformly have a causative role in SEA given the potential for recall bias in its self-reporting [22].

Another limitation of the current risk factors is that formal studies to elucidate their independent association with SEA through statistical analyses have often involved a relatively small number of patients with contradictory results. For example, in a case-control study of patients admitted to a rehabilitation facility following SEA or spinal trauma, those with SEA were more likely to have used IV drugs but were not more likely to have DM or a history of alcohol abuse [23]. Another study involving patients with SEA or spinal subdural abscess found that a history of DM, IVDU, spinal trauma, and degenerative spinal disease were not associated with SEA, while obesity and alcoholism were more predictive [24].

The sheer number of published risk factors also limits their clinical utility in the diagnosis of SEA. Some papers report as many as 50 "risk factors and sources of infection," which often reflect common comorbidities or conditions often associated with $S$. aureus bacteremia, including DM, hemodialysis, HIV infection, heart disease, cancer, alcohol abuse, IVDU, COPD, and soft tissue infections [7, 25, 26]. Further diminishing the utility of risk factors in considering a diagnosis of SEA is their apparent absence in up to $20 \%$ to $50 \%$ of cases [5, 27-29].

\section{Pathophysiology}

3.1. Sources of Infection. SEA develops when microorganisms gain access to the epidural space via hematogenous spread from a distant source such as skin or respiratory or urinary tract; from contiguous foci of infection such as the psoas muscle or vertebra; or by direct inoculation through spinal instrumentation, injection, or catheter placement [3]. Of these mechanisms, hematogenous spread is the most common, accounting for about half of all cases [8], followed by direct spread from a contiguous focus of infection (about one-third); no source is identified in the remaining cases [8]. Interestingly, the location of SEA among patients with IVDU history may correlate with the site of drug injection, with cervical and lumbar spine more likely to be associated with upper and lower extremity sites of injection, respectively [2].

Another potential route of hematogenous infection is the pelvic cavity's venous drainage system, which connects with those of the spine and cranium via the spinal veins and forms Batson's plexus [10]. This valveless venous network may facilitate spread of organisms from pelvic organs (such as the urinary bladder) to the spinal column. More distant sources of infection, such as the oral cavity, should also be considered [30-32], particularly when the source of SEA is not readily apparent.

3.2. Mechanism of Neurological Deficits. Although neurological deficits caused by SEA are often attributed to its direct compression on the spinal cord resulting in ischemia and injury [9], local circulatory disruption due to venous stasis or thrombosis of spinal arteries has also been implicated $[9,12$, 33]. This hypothesis may explain the difficulty in predicting the tempo of neurological complications following symptom onset in SEA, a view that is not universally endorsed, however $[3,22,34]$.

\section{Pathogens}

Although SEA may be caused by a countless number of organisms, Staphylococcus aureus accounts for the majority of cases $(60 \%-90 \%)$ with methicillin-resistant S. aureus (MRSA) accounting for an increasing number [2, 3, 8, 35-37]. Among aerobic Gram-negative bacilli, Escherichia coli often causes SEA in patients with UTI, while Pseudomonas aeruginosa may be the culprit in the setting of IVDU. Other pathogens such as mycobacteria, including Mycobacterium tuberculosis, tend to target immunosuppressed patients, while staphylococcal species other than $S$. aureus and fungi such as Candida species are often associated with spinal instrumentation or injection. SEA caused by an environmental fungus, Exserohilum rostratum, was recently reported in a multistate outbreak involving contaminated corticosteroid injections [38].

\section{Diagnosis}

5.1. Symptoms and Signs. Back pain is the most common presenting symptom of SEA, occurring in $70 \%$ to $100 \%$ of patients $[8,15,27,39]$. The pain tends to be severe and localized with a duration of 1 day to 2 months prior to presentation $[3,8,38]$. Fever is found in about $50 \%$, and back tenderness has been reported in $17 \%$ to $98 \%$ of cases [3, 8, 39-41]. Neurological manifestations, such as motor weakness, radiculopathy, and bladder and bowel dysfunction, have been reported in up to half of the cases [3]. Atypical manifestations of SEA, such as sudden paralysis, abdominal pain, headache, and bowel dysfunction, have also been reported [42-44]. It should be emphasized that the classic triad of back pain, fever, and 
neurological deficits is found in only a minority of patients with SEA [8].

5.2. Laboratory Abnormalities. Leukocytosis has been reported in $60 \%$ to $80 \%$ of patients presenting with SEA $[3,15]$. Serum C-reactive protein (CRP) and erythrocyte sedimentation rate (ESR) have higher sensitivities and are almost uniformly elevated in patients with SEA [3]. Although blood cultures yield an organism in only about half of patients with SEA, they should be obtained routinely as they may guide antibiotic selection when the tissue cultures are not helpful or available [33].

5.3. Radiographic Abnormalities. Gadolinium-enhanced MRI is the radiographic test of choice for the detection of SEA with greater than $90 \%$ sensitivity and specificity $[2,3]$. Some investigators have advocated imaging of the entire spine to exclude noncontiguous SEA in patients with symptom duration of at least one week prior to presentation, the presence of concomitant area of infection outside the spinal region, and when ESR is greater than $95 \mathrm{~mm} / \mathrm{h}$ [45]. In patients with persistent symptoms but an initially unremarkable MRI, repeat testing in 2 to 3 weeks should be considered $[3,38]$. CT myelography is usually not recommended because of its invasive nature and the potential for inadvertent contamination of the subarachnoid space [3]. Computed tomography (CT) scan with intravenous contrast has lower sensitivity, particularly in the early stages of SEA, and should be considered only when MRI cannot be performed $[3,8]$. Nuclear medicine studies, such as technetium and indium isotope scans, have very little role in the diagnosis of SEA due to their suboptimal sensitivity and poor anatomical resolution $[10,15]$.

5.4. Invasive Diagnostic Tests. Once SEA is suspected radiographically, direct sampling of the infected fluid or tissue via image-guided biopsy should be performed to help confirm the diagnosis and direct antimicrobial therapy [3]. In some cases, the need for diagnostic aspiration or sampling may be obviated by isolation of a common etiologic pathogen, such as $S$. aureus, from blood cultures. Lumbar puncture should be avoided in patients with suspected SEA due to the risk of spread of infection into the subarachnoid space [3].

\section{Treatment}

Antibiotics should be administered as soon as cultures from blood and other possible sources of infection have been obtained [1]. Antimicrobial therapy should not be withheld preoperatively in patients with suspected sepsis or neurological symptoms, given the high degree of concordance between blood and abscess cultures and the importance of timely therapy $[3,46]$. Routine empiric coverage of staphylococci (including MRSA), streptococci, and aerobic Gram-negative pathogens such as a combination of vancomycin with either piperacillin-tazobactam or a third- or fourth-generation cephalosporin (such as ceftazidime or cefepime) is often recommended $[3,8,9]$. Once the causative pathogen has been isolated, deescalation of therapy is advised. Patients at risk of fungal SEA should also receive an antifungal agent such as voriconazole or amphotericin B [38].

In addition to antimicrobial therapy, prompt surgery is indicated in most cases of SEA [3, 8, 27, 40, 46, 47]. Successful treatment of SEA with medical therapy alone in selected patients has also been reported [27, 40, 46, 48-51]. However, caution is advised when interpreting these reports because of their observational nature and the potential for selection of less severely ill patients for medical therapy alone [3]. A recent review article concluded that most studies advocate for early (within 24 hours of diagnosis) surgery due to high failure rates and a significant risk of morbidity (22\% for permanent paralysis) and mortality (3\% to $25 \%$ ) with nonoperative treatment alone [47]. In contrast, medical therapy alone may be favored in patients with panspinal SEA involvement or those with complete paresis for 72 hours or more or when surgery is deemed too risky $[3,15,40,46]$.

Recommendations for the duration of antibiotic therapy vary widely from 4 weeks to 16 weeks depending on many factors, including comorbidities and concurrent presence of vertebral osteomyelitis [3]. Although the optimal duration of parenteral antibiotics is not always defined, most patients receive at least 2 to 4 weeks of therapy when vertebral osteomyelitis is not suspected [3]. Patients with M. tuberculosisassociated SEA should receive 6 months to 1 year of appropriate therapy (e.g., isoniazid and rifampin). Whenever possible, infected indwelling spinal hardware (such as a spinal stimulator or catheter) should be removed. Close monitoring for signs and symptoms of relapse after completion of antimicrobial therapy is essential in the management of SEA. Of note, one study found a treatment failure rate of $28 \%$ in patients with SEA caused by MRSA [52].

\section{Prognosis}

The outcome of SEA is often assessed based on mortality and recovery from neurological deficits [3]. Although the mortality rate has fallen significantly from $80 \%$ in the preantibiotic era to $2 \%$ to $20 \%$ in recent decades, many patients continue to die from this condition $[3,8,53]$. Death is generally due to overwhelming sepsis and typically occurs in patients with multiple comorbidities [3].

About one-third of survivors may have a poor neurological outcome often due to diagnostic delays [3]. In fact, a patient's ultimate neurological outcome correlates strongly with the severity and duration of neurological deficits prior to surgery [3]. The presence of spinal cord-related deficits upon presentation may also be a risk factor for failure of medical therapy [54]. These findings underscore the importance of diagnosis of SEA before neurological complications develop.

\section{Potential Strategies for Earlier Diagnosis}

A major obstacle to the timely diagnosis of SEA is the nonspecificity of its signs and symptoms. In addition, as previously discussed, for the clinician evaluating a patient with severe back pain, the utility of published risk factors is limited due to their uncertain role in SEA causation, seemingly 
countless number, and absence in a significant proportion of patients who are ultimately diagnosed with SEA.

Only a few studies have examined specific strategies for reducing delays in the diagnosis of SEA. A single-center study of routine MRI screening in emergency room patients suspected of having SEA yielded a diagnosis of SEA in less than $7 \%$ of patients [41] despite more than half of the patients having a history of IVDU. This study questions the costeffectiveness of routine MRI for back pain, even among patients considered at high risk for SEA. Of note, none of the patient demographics studied in this study, including history of IVDU, was helpful in distinguishing patients who had SEA from those who did not.

Another single-center study evaluated the impact of a decision guide on diagnostic delays of SEA in patients presenting to an ED with back pain [1]. One or more risk factors for SEA were present in $100 \%$ of patients ultimately diagnosed with SEA, as compared to $23 \%$ of controls. In addition, an elevated ESR or CRP was found in $100 \%$ and $87 \%$ of SEA cases, respectively. A decision-making guide evaluating patients with progressive neurological deficits or elevation of either the ESR or the CRP-combined with the presence of one or more prespecified risk factors for SEA, fever, or radicular pain-was suggested. For all other patients without an obvious source of pain, discharge with follow-up was recommended. Although a decrease in diagnostic delays was observed following adoption of the algorithm compared to historical data, the presence of a risk factor in 100\% of cases (compared to frequently lower rates in other studies), the reported inconsistent application of the algorithm by providers, and lack of an active surveillance system for followup of patients with risk factors but no ESR and CRP on file made interpretation of the data problematic [1].

Another potential strategy for early detection of SEA consists of obtaining MRI in all patients with severe back pain. However, the feasibility and cost-effectiveness of this approach may be questioned in the current era of stewardship of healthcare resources [41].

We propose an alternative strategy in the evaluation of patients with severe back pain which uses a simple algorithm (Figure 1). This algorithm is based on the pathophysiology of SEA, its often unpredictable clinical course, the high sensitivities of ESR and CRP in this condition, and a limited number of risk factors for which a direct role in the causation of SEA can be easily invoked (i.e., S. aureus bacteremia, contiguous focus of infection, and spinal injection/instrumentation). Accordingly, we recommend that patients with severe back pain and progressive neurological deficits undergo emergent MRI, or a CT if MRI is contraindicated. In the absence of progressive neurological deficits, those with recent $S$. aureus bacteremia or spinal injection/instrumentation should undergo urgent (within $24 \mathrm{~h}$ ) MRI. In all other patients, ESR and CRP should be obtained, and if either is elevated, MRI should be considered, with its timing contingent upon the overall assessment of the patient, severity of pain, and the likelihood of noninfectious explanations for the back pain. If both ESR and CRP are normal, SEA would be much less likely and further workup for noninfectious causes may be pursued as appropriate.

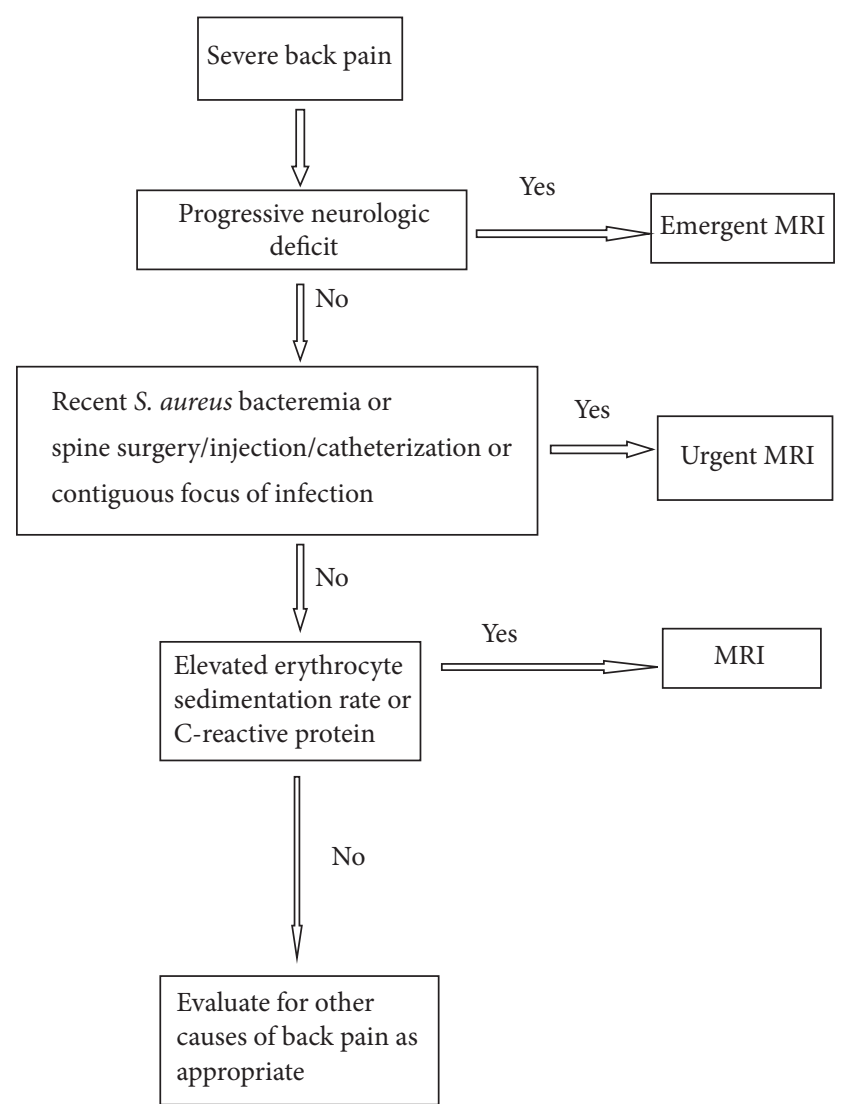

FIgURE 1: The proposed algorithm for SEA diagnosis in patients presenting with severe back pain.

We further suggest that, in patients without back pain but at high risk of SEA because of a recent bacteremic illness (particularly caused by $S$. aureus), routine examination for spinal tenderness should be considered. This strategy is not dissimilar to searching for signs of endocarditis, such as new cardiac murmurs or embolic lesions, in patients with $S$. aureus bacteremia [55]. Of note, in a recent study of patients with MRSA bacteremia, the rate of SEA was the same as that of endocarditis (4\% each) [56].

\section{Conclusion}

SEA is an uncommon but potentially devastating condition that continues to challenge the diagnostic skills of many clinicians. Reliance on published risk factors to help reduce diagnostic delays in SEA is limited by their seemingly countless number and their absence in a significant proportion of patients. More practical and feasible approaches to earlier diagnosis of SEA are sorely needed.

\section{Competing Interests}

The authors have no financial disclosures to declare and no competing interests to report. 


\section{References}

[1] D. P. Davis, A. Salazar, T. C. Chan, and G. M. Vilke, "Prospective evaluation of a clinical decision guideline to diagnose spinal epidural abscess in patients who present to the emergency department with spine pain," Journal of Neurosurgery: Spine, vol. 14, no. 6, pp. 765-770, 2011.

[2] E. S. Nussbaum, D. Rigamonti, H. Standiford, Y. Numaguchi, A. L. Wolf, and W. L. Robinson, "Spinal epidural abscess: a report of 40 cases and review," Surgical Neurology, vol. 38, no. 3, pp. 225-231, 1992.

[3] P. Sendi, T. Bregenzer, and W. Zimmerli, "Spinal epidural abscess in clinical practice," Quarterly Journal of Medicine, vol. 101, no. 1, pp. 1-12, 2008.

[4] K. Kan and M. Mehta, Microbiology for Surgical Infections: Diagnosis, Prognosis and Treatment, Spinal Epidural Abscesses, 2014.

[5] M. Shousha and H. Boehm, "Surgical treatment of cervical spondylodiscitis: a review of 30 consecutive patients," Spine, vol. 37, no. 1, pp. E30-E36, 2012.

[6] I. Strauss, N. Carmi-Oren, A. Hassner et al., "Spinal epidural abscess: in search of reasons for increased incidence," The Israel Medical Association Journal, vol. 15, no. 9, pp. 560-564, 2013.

[7] E. Reihsaus, H. Waldbaur, and W. Seeling, "Spinal epidural abscess: a meta-analysis of 915 patients," Neurosurgical Review, vol. 23, no. 4, pp. 175-204, 2000.

[8] R. O. Darouiche, "Spinal epidural abscess," New England Journal of Medicine, vol. 355, no. 19, pp. 2012-2020, 2006.

[9] G. Pradilla, G. P. Ardila, W. Hsu, and D. Rigamonti, "Epidural abscesses of the CNS," The Lancet Neurology, vol. 8, no. 3, pp. 292-300, 2009.

[10] W. Y. Cheung and K. D. K. Luk, "Pyogenic spondylitis," International Orthopaedics, vol. 36, no. 2, pp. 397-404, 2012.

[11] R. M. Duarte and A. R. Vaccaro, "Spinal infection: State of the art and management algorithm," European Spine Journal, vol. 22, no. 12, pp. 2787-2799, 2013.

[12] N. H. Shah and K. L. Roos, "Spinal epidural abscess and paralytic mechanisms," Current Opinion in Neurology, vol. 26, no. 3, pp. 314-317, 2013.

[13] K. Rosc-Bereza, M. Arkuszewski, E. Ciach-Wysocka, and M. Boczarska-Jedynak, "Spinal epidural abscess: common symptoms of an emergency condition," Neuroradiology Journal, vol. 26, no. 4, pp. 464-468, 2013.

[14] P. Krishnamohan and J. R. Berger, "Spinal epidural abscess," Current Infectious Disease Reports, vol. 16, no. 11, p. 436, 2014.

[15] M. Tompkins, I. Panuncialman, P. Lucas, and M. Palumbo, "Spinal epidural abscess," Journal of Emergency Medicine, vol. 39, no. 3, pp. 384-390, 2010.

[16] M. Kundi, "Causality and the interpretation of epidemiologic evidence," Environmental Health Perspectives, vol. 114, no. 7, pp. 969-974, 2006.

[17] J. D. Beck, "Risk revisited," Community Dentistry and Oral Epidemiology, vol. 26, no. 4, pp. 220-225, 1998.

[18] H. C. Kraemer, A. E. Kazdin, D. R. Offord, R. C. Kessler, P. S. Jensen, and D. J. Kupfer, "Coming to terms with the terms of risk," Archives of General Psychiatry, vol. 54, no. 4, pp. 337-343, 1997.

[19] Y. Erşahin, "Spinal epidural abscess: a meta-analysis of 915 patients," Neurosurgical Review, vol. 24, no. 2-3, p. 156, 2001.

[20] K. Middleton and D. E. Fish, "Lumbar spondylosis: clinical presentation and treatment approaches," Current Reviews in Musculoskeletal Medicine, vol. 2, no. 2, pp. 94-104, 2009.
[21] M. F. Lin, Y. J. Lau, B. S. Hu, Z. Y. Shi, and Y. H. Lin, "Pyogenic psoas abscess: analysis of 27 cases," Journal of microbiology, immunology, and infection, vol. 32, no. 4, pp. 261-268, 1999.

[22] S. Kastenbauer, H.-W. Pfister, and W. M. Scheld, "Epidural abscess," in Infections of the Central Nervous System, W. M. Schedl, R. J. Whitely, and C. M. Marra, Eds., pp. 509-522, Lippincott Williams \& Wilkins, Philadelphia, Pa, USA, 2004.

[23] R. D. Zafonte, J. H. Ricker, R. A. Hanks, D. L. Wood, A. Amin, and L. Lombard, "Spinal epidural abscess: study of early outcome," Journal of Spinal Cord Medicine, vol. 26, no. 4, pp. 345351, 2003.

[24] M. Angsuwat, B. Kavar, and A. J. Lowe, "Early detection of spinal sepsis," Journal of Clinical Neuroscience, vol. 17, no. 1, pp. 59-63, 2010.

[25] K. B. Laupland, D. L. Church, M. Mucenski, L. R. Sutherland, and H. D. Davies, "Population-based study of the epidemiology of and the risk factors for invasive Staphylococcus aureus infections," Journal of Infectious Diseases, vol. 187, no. 9, pp. 1452-1459, 2003.

[26] C. Hernandez, N. Cobos-Trigueros, C. Feher et al., "Community-onset bacteraemia of unknown origin: clinical characteristics, epidemiology and outcome," European Journal of Clinical Microbiology and Infectious Diseases, vol. 33, no. 11, pp. 19731980, 2014.

[27] A. R. Patel, T. B. Alton, R. J. Bransford, M. J. Lee, C. B. Bellabarba, and J. R. Chapman, "Spinal epidural abscesses: risk factors, medical versus surgical management, a retrospective review of 128 cases," Spine Journal, vol. 14, no. 2, pp. 326-330, 2014.

[28] P. Sørensen, "Spinal epidural abscesses: conservative treatment for selected subgroups of patients," British Journal of Neurosurgery, vol. 17, no. 6, pp. 513-518, 2003.

[29] M. Soehle and T. Wallenfang, "Spinal epidural abscesses: clinical manifestations, prognostic factors, and outcomes," Neurosurgery, vol. 51, no. 1, pp. 79-87, 2002.

[30] S. M. Patel, J. H. Mo, M. T. Walker, B. Adley, and G. A. Noskin, "Epidural abscess and osteomyelitis due to Actinobacillus actinomycetemcomitans," Diagnostic Microbiology and Infectious Disease, vol. 50, no. 4, pp. 283-285, 2004.

[31] S. Korfias, G. A. Alexiou, E. Vlachakis, and D. E. Sakas, "Cervical epidural abscess of odontogenic origin," Neurological Sciences, vol. 36, no. 6, pp. 1017-1018, 2015.

[32] S. I. Goolamali, M. T. Carulli, and U. M. Davies, "Spinal abscess and mitral valve endocarditis secondary to asymptomatic fusobacterium-induced dental abscess," Journal of the Royal Society of Medicine, vol. 99, no. 7, pp. 368-369, 2006.

[33] R. O. Darouiche, R. J. Hamill, S. B. Greenberg, S. W. Weathers, and D. M. Musher, "Bacterial spinal epidural abscess. Review of 43 cases and literature survey," Medicine, vol. 71, no. 6, pp. 369$385,1992$.

[34] A. Tuchman, M. Pham, and P. C. Hsieh, "The indications and timing for operative management of spinal epidural abscess: literature review and treatment algorithm," Neurosurgical Focus, vol. 37, no. 2, article E8, 2014.

[35] G. M. Ghobrial, S. Beygi, M. J. Viereck et al., "Timing in the surgical evacuation of spinal epidural abscesses," Neurosurgical Focus, vol. 37, no. 2, article E1, 2014.

[36] W. C. Ziai and J. J. Lewin, "Update in the diagnosis and management of central nervous system infections," Neurologic Clinics, vol. 26, no. 2, pp. 427-468, 2008. 
[37] D. E. Connor Jr., P. Chittiboina, G. Caldito, and A. Nanda, "Comparison of operative and nonoperative management of spinal epidural abscess: a retrospective review of clinical and laboratory predictors of neurological outcome. Clinical article," Journal of Neurosurgery: Spine, vol. 19, no. 1, pp. 119-127, 2013.

[38] C. A. Kauffman, P. G. Pappas, and T. F. Patterson, "Fungal infections associated with contaminated methylprednisolone injections," The New England Journal of Medicine, vol. 368, no. 26, pp. 2495-2500, 2013.

[39] O. Adogwa, I. O. Karikari, K. R. Carr et al., "Spontaneous spinal epidural abscess in patients 50 years of age and older: a 15-year institutional perspective and review of the literature: clinical article," Journal of Neurosurgery: Spine, vol. 20, no. 3, pp. 344$349,2014$.

[40] W. T. Curry Jr., B. L. Hoh, S. Amin-Hanjani, and E. N. Eskandar, "Spinal epidural abscess: clinical presentation, management, and outcome," Surgical Neurology, vol. 63, no. 4, pp. 364-371, 2005.

[41] M. El Sayed and M. D. Witting, "Low yield of ED magnetic resonance imaging for suspected epidural abscess," The American Journal of Emergency Medicine, vol. 29, no. 9, pp. 978-982, 2011.

[42] M. L. Noy and S. George, "Unusual presentation of a spinal epidural abscess," BMJ Case Reports, vol. 2012, 2012.

[43] A. Prakash, S. Kubba, N. P. Singh et al., "Tuberculous epidural abscess: an unusual presentation," Indian Journal of Tuberculosis, vol. 51, pp. 157-158, 2004.

[44] A. A. Bremer and R. O. Darouiche, "Spinal epidural abscess presenting as intra-abdominal pathology: a case report and literature review," Journal of Emergency Medicine, vol. 26, no. 1, pp. 51-56, 2004.

[45] K. L. Ju, S. D. Kim, R. Melikian, C. M. Bono, and M. B. Harris, "Predicting patients with concurrent noncontiguous spinal epidural abscess lesions," Spine Journal, vol. 15, no. 1, pp. 95-101, 2015.

[46] D. Rigamonti, L. Liem, P. Sampath et al., "Spinal epidural abscess: contemporary trends in etiology, evaluation, and management," Surgical Neurology, vol. 52, no. 2, pp. 189-197, 1999.

[47] N. E. Epstein, "Timing and prognosis of surgery for spinal epidural abscess: a review," Surgical Neurology International, vol. 6, supplement 19, pp. S475-S486, 2015.

[48] T. B. Alton, A. R. Patel, R. J. Bransford, C. Bellabarba, M. J. Lee, and J. R. Chapman, "Is there a difference in neurologic outcome in medical versus early operative management of cervical epidural abscesses?” Spine Journal, vol. 15, no. 1, pp. 10-17, 2015.

[49] K. Savage, P. D. Holtom, and C. G. Zalavras, "Spinal epidural abscess: early clinical outcome in patients treated medically," Clinical Orthopaedics and Related Research, no. 439, pp. 56-60, 2005.

[50] F. Siddiq, A. Chowfin, R. Tight, A. E. Sahmoun, and R. A. Smego Jr., "Medical vs surgical management of spinal epidural abscess," Archives of Internal Medicine, vol. 164, no. 22, pp. 2409-2412, 2004.

[51] G. Sengul, A. Akar, F. Alper, and H. Uslu, "Nonsurgically treated cervical brucellar epidural abscess causing spinal cord compression," Journal of Clinical Neuroscience, vol. 15, no. 12, pp. 1411-1414, 2008.

[52] J. C. Dombrowski and L. G. Winston, "Clinical failures of appropriately-treated methicillin-resistant Staphylococcus aureus infections," Journal of Infection, vol. 57, no. 2, pp. 110-115, 2008.

[53] S. Grewal, G. Hocking, and J. A. W. Wildsmith, "Epidural abscesses," British Journal of Anaesthesia, vol. 96, no. 3, pp. 292-302, 2006.
[54] D. E. Connor, P. Chittiboina, G. Caldito, and A. Nanda, "Comparison of operative and nonoperative management of spinal epidural abscess: a retrospective review of clinical and laboratory predictors of neurological outcome," Journal of Neurosurgery: Spine, vol. 19, no. 1, pp. 119-127, 2013.

[55] S. S. Lewis and D. J. Sexton, "Metastatic complications of bloodstream infections in hemodialysis patients," Seminars in Dialysis, vol. 26, no. 1, pp. 47-53, 2013.

[56] T. Horino, F. Sato, Y. Hosaka et al., "Predictive factors for metastatic infection in patients with bacteremia caused by methicillin-sensitive staphylococcus aureus," American Journal of the Medical Sciences, vol. 349, no. 1, pp. 24-28, 2014. 


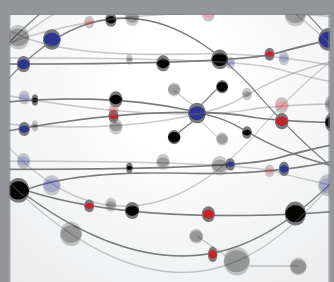

The Scientific World Journal
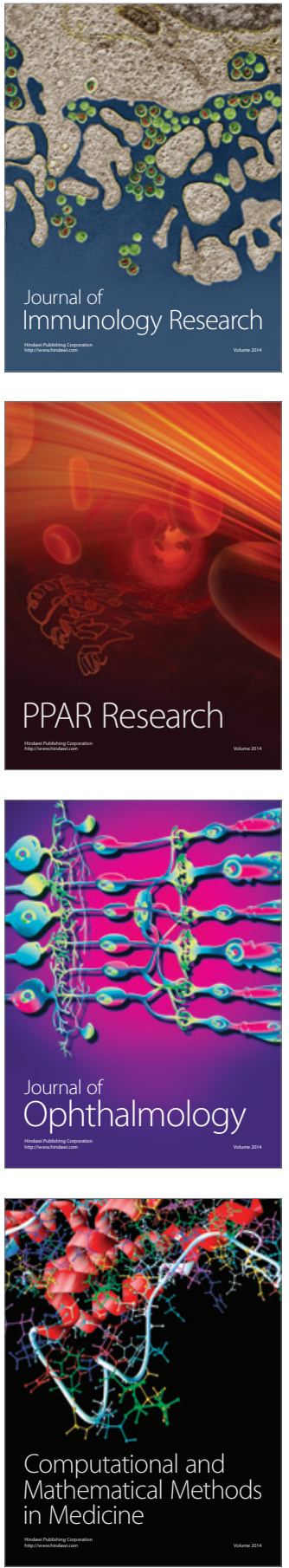

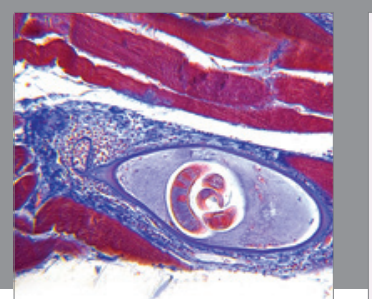

Gastroenterology Research and Practice

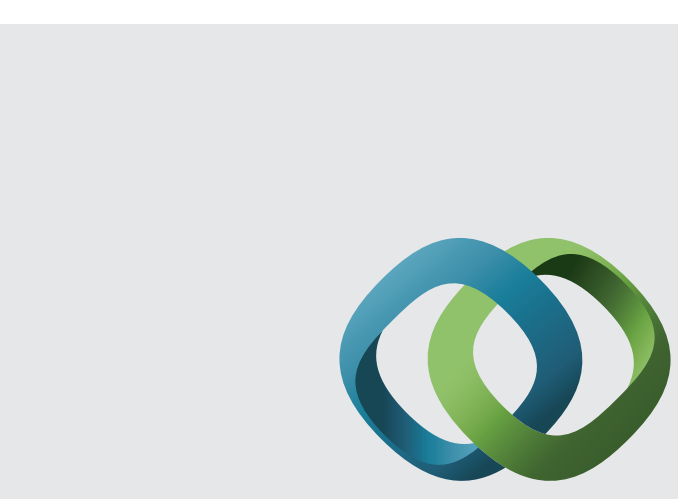

\section{Hindawi}

Submit your manuscripts at

http://www.hindawi.com
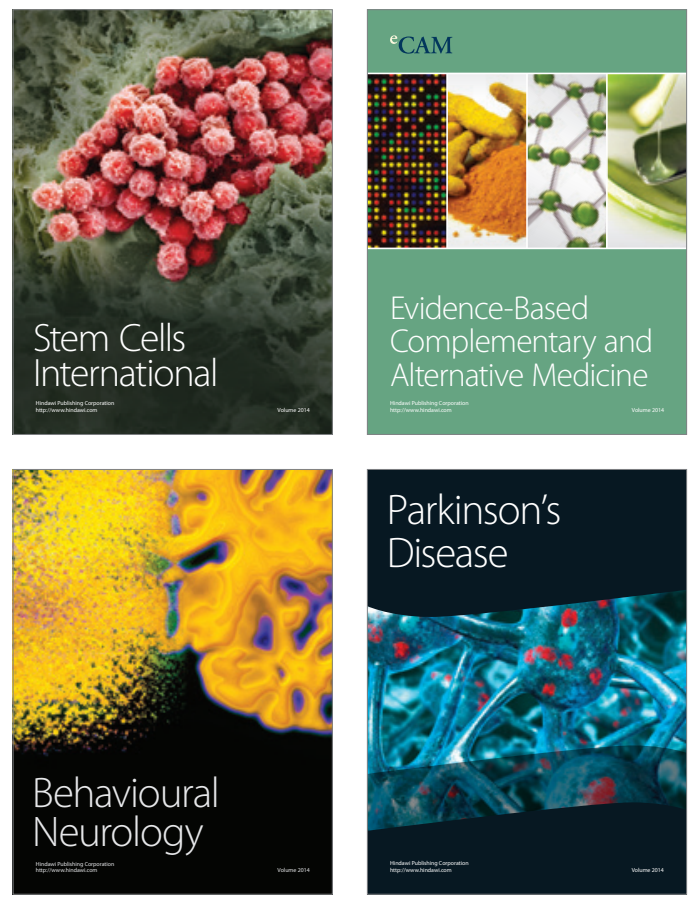
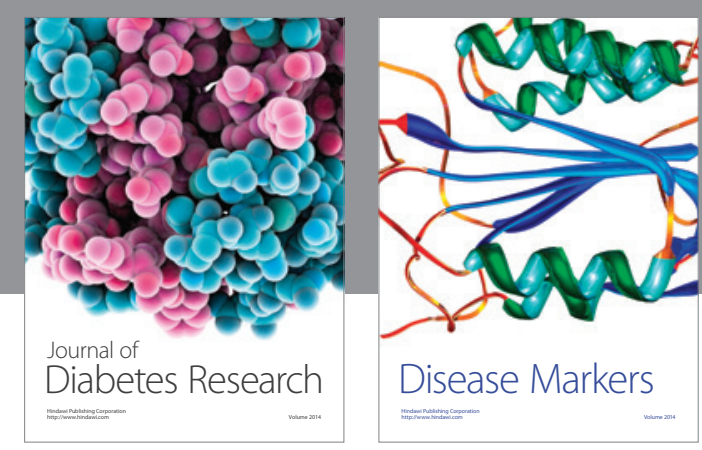

Disease Markers
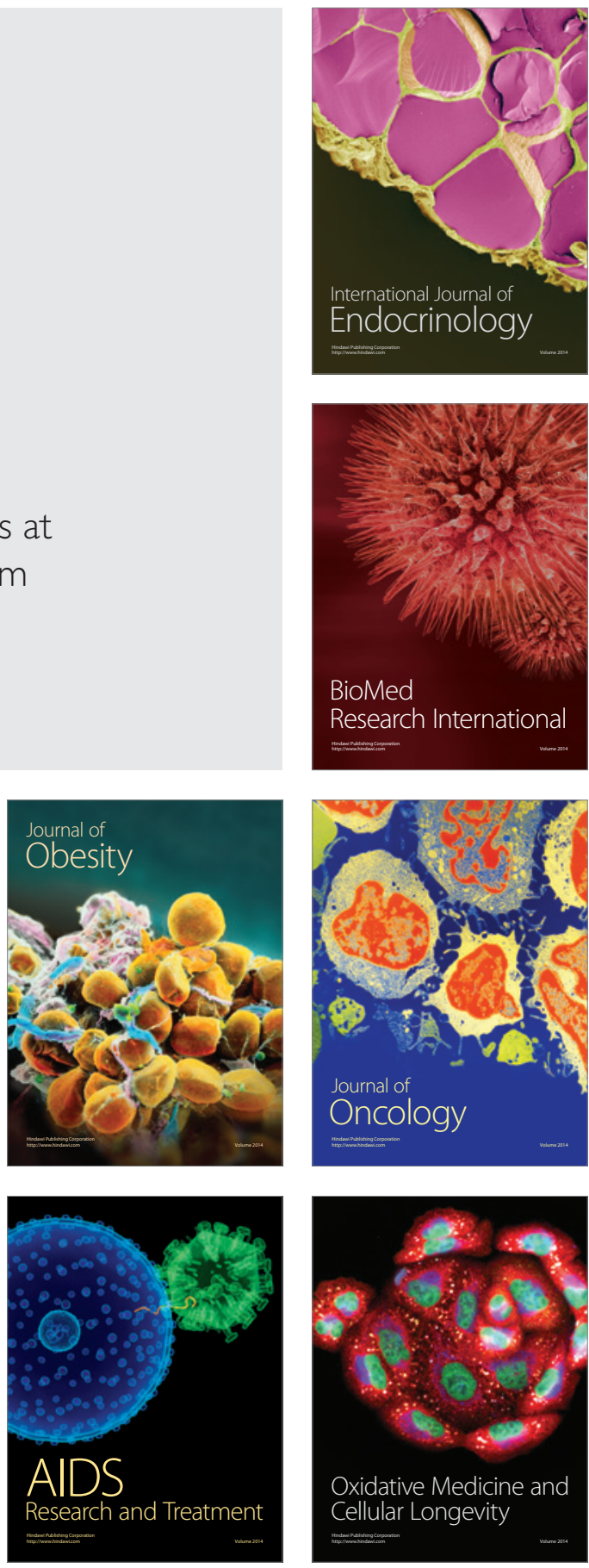